C-A/AP/\#218

September 2005

\title{
CW Mode of Operation of a Proton FFAG Accelerator
}

Alessandro G. Ruggiero

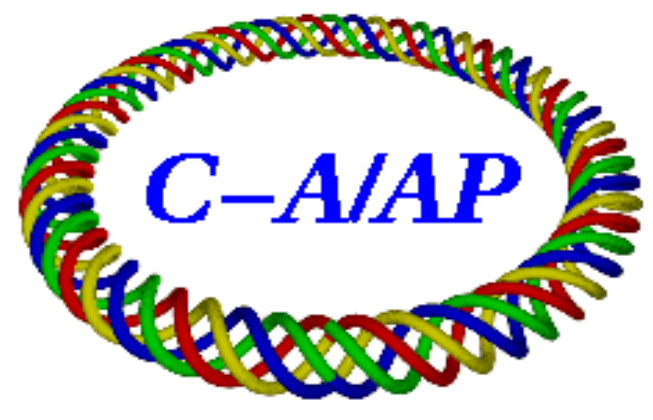

Collider-Accelerator Department Brookhaven National Laboratory Upton, NY 11973 


\title{
CW Mode of Operation of a Proton FFAG Accelerator
}

\author{
Alessandro G. Ruggiero \\ Brookhaven National Laboratory \\ August 30, 2005
}

In a previous note [1] we have studied a Fixed-Field Alternating-Gradient (FFAG) Accelerator for a Proton Driver with $1.0 \mathrm{GeV}$ kinetic energy and average beam power of $10 \mathrm{MWatt}$. The mode of operation discussed for that facility was a pulsed mode with a repetition rate of $1 \mathrm{kHz}$, probably the highest feasible for such an accelerator with a single-turn beam circulating at any time. The duty cycle is then modest, determined by the length of the extracted beam pulse length of $0.77 \mu$ s corresponding to a circumference of $201.8 \mathrm{~m}$. The duty factor is $0.077 \%$, useful for several applications like, for instance, Pulsed Spallation Neutron Sources. But other applications would still require possibly a Continuous Wave (CW) mode of operation with 100\% duty cycle. Such factor can be obtained in Cyclotrons [2] and Super-Conducting Linacs (SCL) [3]. It would be highly desirable if a $\mathrm{CW}$ mode of operation can also be made possible in an FFAG Accelerator.

In an FFAG accelerator the beam spirals from an internal orbit (injection) to an external orbit (extraction) and comes to occupy in turn different intermediate orbits. Each orbit corresponds to a radial displacement and to a beam momentum. All trajectories traverses the same RF cavity system located in one or few discrete locations, and all particles, whatever is their location and momentum, will have to cross the RF cavities in phase for an optimal energy gain. In the pulsed mode of operation only one orbit is occupied at the time by the beam, and energy gain by cavity traversal does not present a problem, as already described in [1]. But if one desires a $\mathrm{CW}$ mode of operation there will be beam on every orbit and simultaneous energy gain for all beam turns becomes problematic.

This is not a problem for light particles like electrons and muons because they have for all purposes a speed very close to that of light. It is then sufficient that the lattice of the FFAG ring operates with the beam energy matching the transition energy. In this case [4] isochronous condition is easily satisfied since all particles travel between cavity crossings at the same time interval. But isochronous condition is not easily satisfied for low energy protons because of the changing of their velocity during acceleration.

The revolution period of a particle with momentum $\mathrm{p}$ is given by $\mathrm{T}(\mathrm{p})=\mathrm{C}(\mathrm{p})$ / $c \beta(p)$ where $C(p)$ and $c \beta(p)$ are respectively the path length per turn and the velocity of that particle. By differentiating around $\mathrm{T}_{0}, \mathrm{C}_{0}$ and $\beta_{0}$ corresponding to a reference momentum value $\mathrm{p}_{0}$ (that we assume at injection), we have

$$
\Delta \mathrm{T} / \mathrm{T}_{0} \quad=\quad \Delta \mathrm{C} / \mathrm{C}_{0}-\Delta \beta / \beta_{0}
$$

and in linear approximation with respect to the momentum variation $\Delta \mathrm{p} / \mathrm{p}_{0}$ 


$$
\Delta \mathrm{T} / \mathrm{T}_{0} \quad=\quad\left(\alpha_{\mathrm{p}}-\gamma^{-2}\right) \Delta \mathrm{p} / \mathrm{p}_{0}
$$

where $\alpha_{\mathrm{p}}$ is the momentum compaction factor and $\gamma$ the energy relativistic factor.

There are thus two contributions: the first is the path length difference and the second the velocity difference on the same orbit. Isochronous condition is achieved when the two contributions cancel each other. Figure 1 is the plot of the path length variation $\Delta \mathrm{C} / \mathrm{C}_{0}$ required for isochronous condition versus the momentum deviation $\Delta \mathrm{p} / \mathrm{p}_{0}$ for the Proton Driver discussed in [1]. Though the path length variation required may sound excessive, nevertheless magnets can be shaped in length so to create the required path length difference (spiral FFAG). Prescriptions to design the shape of the magnet were already worked out a half a century ago [5] and actually used in the design of FFAG accelerators proposed about two decades ago for Spallation Neutron Sources [6]. Unfortunately the approach requires considerable large aperture magnets. Isochronous condition is satisfied in Cyclotrons though at a cost of large size magnets [2], and is certainly not an issue in SCL [3].

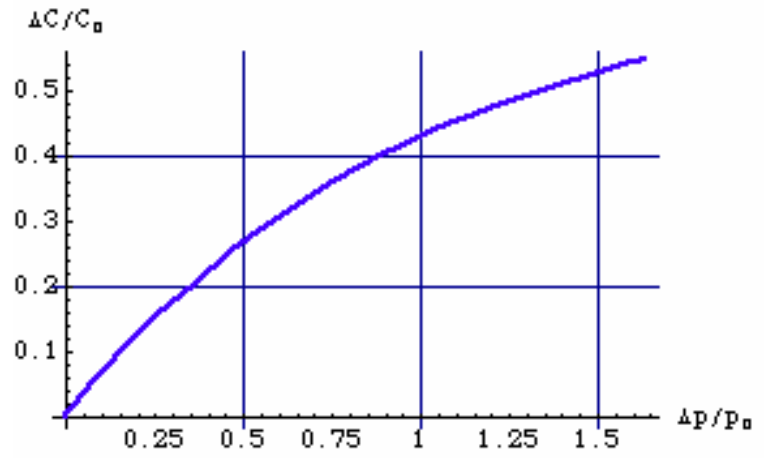

Figure 1. Path Length Variation versus Momentum Deviation for Isochronous Condition

An alternative method that we propose here is based on the Harmonic Number Jump. This makes use of a constant high RF frequency for acceleration without any need of modulation and hence of ferrite. When a particle on a certain trajectory crosses the $\mathrm{RF}$ cavity it receives an energy gain $\Delta \mathrm{E}$ such that the revolution period $\mathrm{T}$ changes by an amount $\Delta \mathrm{T}$ to arrive at the next turn at the same cavity location still in phase but displaced by one RF wavelength, that is on the earlier RF bucket. This is equivalent to a Harmonic Number Jump given by the condition $\Delta \mathrm{T} / \mathrm{T}_{0}=-1 / \mathrm{h}$, where $\mathrm{h}$ is the RF harmonic number. The required RF voltage that satisfies this condition is

$$
\mathrm{eV}=\Delta \mathrm{E}=\mathrm{E}_{0} \beta^{2} \gamma^{3} / \mathrm{h}\left(1-\alpha_{\mathrm{p}} \gamma^{2}\right)
$$

where for a typical compact Non-Scaling Lattice $\alpha_{\mathrm{p}}=\gamma_{\mathrm{T}}{ }^{-2}$ is essentially constant across the momentum aperture. Since $\gamma_{\mathrm{T}}>\gamma$ the $\alpha_{\mathrm{p}} \gamma^{2}-$ term at the denominator can be ignored. Figure 2 shows the variation of the $\alpha_{\mathrm{p}} \gamma^{2}$ - term over the entire acceleration cycle of the Proton Driver of Ref. [1].

For the same case of the Proton Driver we have estimated the required RF voltage according to Eq. (3) during the acceleration cycle, and plotted it in Figure 3 for different $\mathrm{RF}$ frequencies. Figure 4 shows the corresponding Harmonic Number $\mathrm{h}$ variation during the same cycle. Inspection of Eq. (3) shows that the required RF voltage decreases linearly with the RF frequency as it can indeed observed from the curves of Figure 3. Because of the considerable higher voltage, the acceleration period is now considerably 


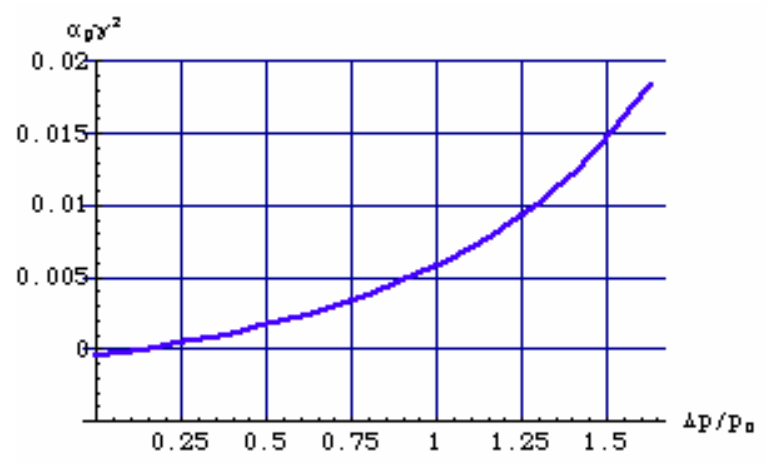

Figure 2. Plot of $\alpha_{\mathrm{p}} \gamma^{2}$ versus Momentum Deviation shortened and is 87,175 and 350 revolutions respectively for 201.25, 402.5 and $805 \mathrm{MHz}$, compared to 667 revolutions in the pulsed mode of operation [1] at constant harmonic number $\mathrm{h}=36$.

A good choice is the frequency of MHz. With superconducting cavities, a modular cell is about $15 \mathrm{~cm}$ long and three of them can be easily accommodated in the $1.25 \mathrm{~m}$ long drift

that separates the FDF triplets that make the FFAG ring [1]. A cavity with three cells can easily sustain a voltage of 2 MVolt, including depletion from a transit time factor, so that a total of only 5 of such cavities are needed to achieve the required peak voltage of 10 MVolt.
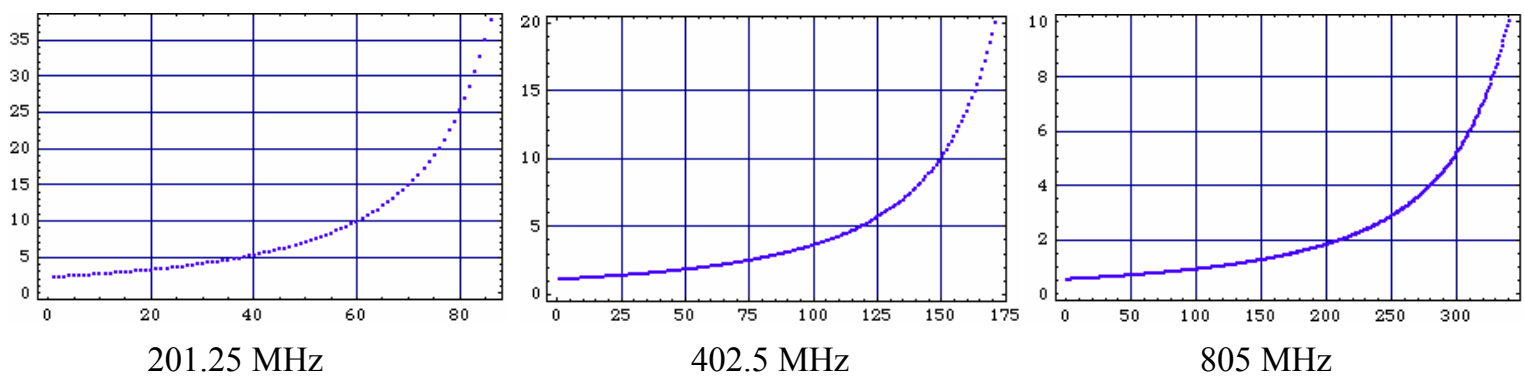

Figure 3. Energy Gain $\Delta \mathrm{E}(\mathrm{MeV} / \mathrm{turn})$ versus Turn Number for different RF Frequencies

The cavity RF voltage will have therefore to vary across the radial aperture with a
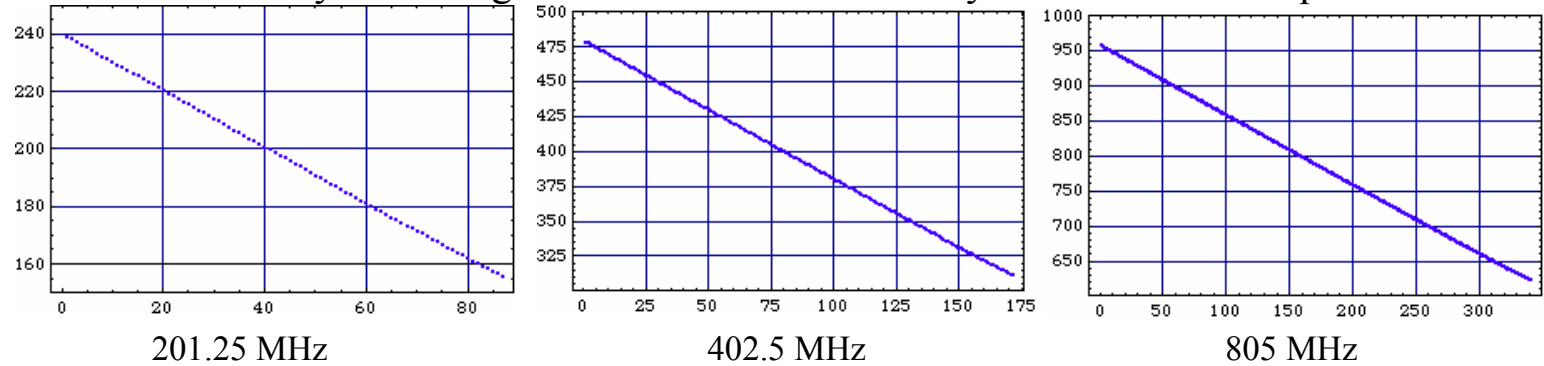

Figure 4. Harmonic Number h Change versus Turn Number

waveform matching the curves of Figure 3. Figure 5 shows the radial displacement $x_{p}$ turn after turn during the entire acceleration cycle for the case of $805 \mathrm{MHz}$. To be noted is the similarity in shape between the curves of Figures 3 and 5. Therefore we plotted directly the required energy gain $\Delta \mathrm{E}$ versus the radial displacement $\mathrm{x}_{\mathrm{p}}$ in Figure 6 . The resulting curve is the actual longitudinal RF field profile required across the cavity aperture. It is seen that the profile is practically linear with radial position. 


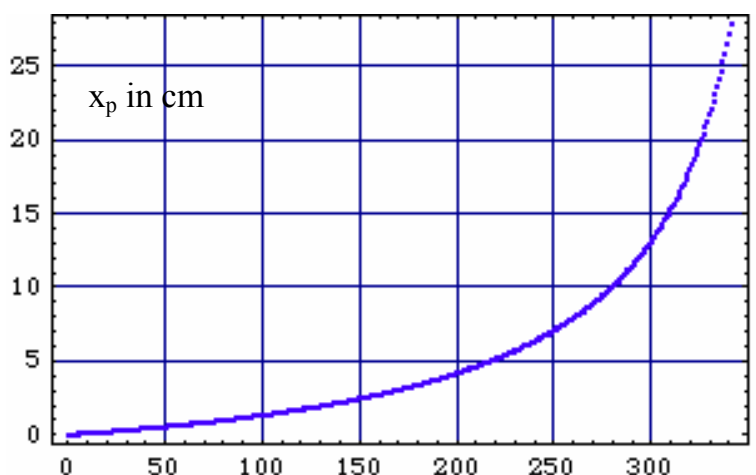

Figure 5. Beam Radial Position vs. Number of Turns

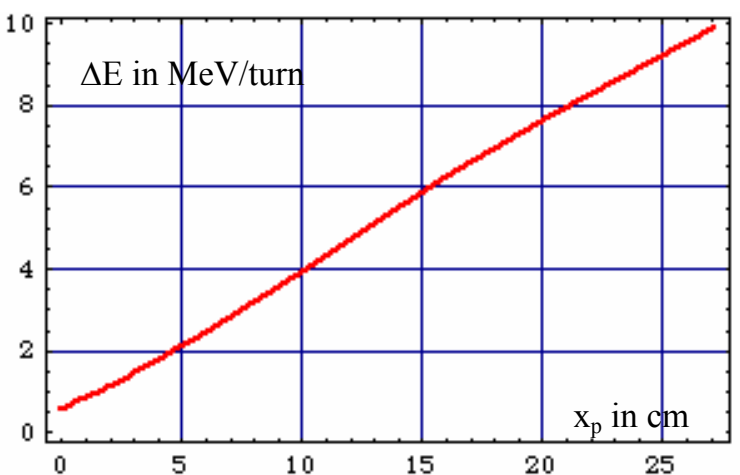

Figure 6. Energy Gain vs. Radial Position

The design of a cavity cell that reproduces the longitudinal field profile of Figure 6 is problematic but not impossible. In the pill-box approximation, denoting with $\mathrm{R}$ the major transverse radius of the cavity, the longitudinal electric field varies with radius $r$ according to the Bessel function $\mathrm{J}_{0}(2.405 \mathrm{r} / \mathrm{R})$ where $\mathrm{r}=0$ corresponds to the axis of the cavity. With the translation of coordinates $r=R-x_{p}$ it is seen that the Bessel function $J_{0}$ approximates the curve of Figure 6 at least in the ascending part. The beam is injected at $x_{p}=0 \mathrm{~cm}$ where the required field is minimum, and accelerated toward $x_{p}=27 \mathrm{~cm}$ where top energy is reached and the beam extracted. The cavity diameter is thus at least twice as large as the beam radial extension during acceleration $(27 \mathrm{~cm})$. A reasonable choice is $\mathrm{R}$ $=35 \mathrm{~cm}$. The maximum value of the longitudinal field is at $\mathrm{x}_{\mathrm{p}}=35 \mathrm{~cm}$ which corresponds to the $\mathrm{r}=0$ axis of the cavity. The cavity is thus transversally offset by a corresponding amount as shown in Figure 7. The maximum value of the field is outside the beam range and likely approaches a value of $12 \mathrm{MVolt}$.

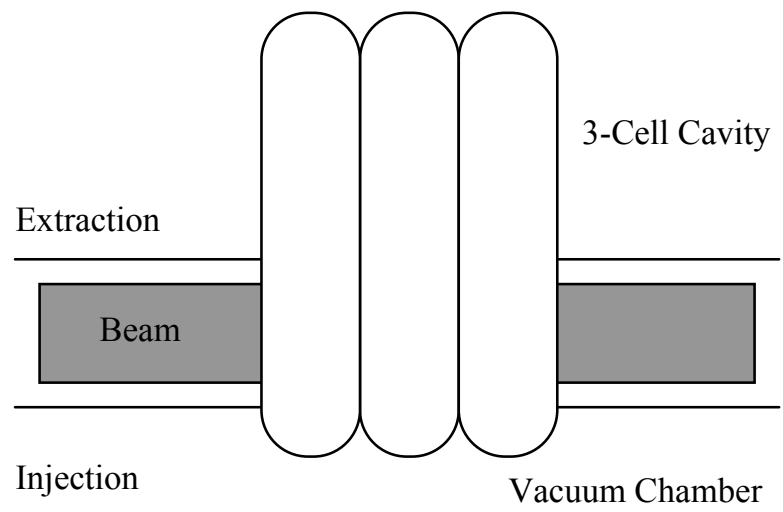

Figure 7. RF Cavity Arrangement with respect to Circulating Beam
The advantage of operating the FFAG accelerator in $\mathrm{CW}$ mode, provided the Harmonic Number Jump method is demonstrated feasible in practice, is that a single beam turn has now a considerably lower intensity. For instance, in the same example of the Proton Driver described in [1], for the same average beam power of $10 \mathrm{MW}$, there are just only $3 \times 10^{11}$ protons circulating on a single turn compared to $6.25 \times 10^{13}$ in the pulsed mode. If the turns are sufficiently differentiated from each other, the local space-charge effects will cause a more modest tune

depression $(\sim 0.1)$ at much reduced beam emittance that again in the same example of Proton Driver is now only $3 \pi \mathrm{mm}$-mrad compared to $150 \pi \mathrm{mm}$-mrad in the pulsed mode. Nevertheless since there are several turns adjacent to each other, the beam shape is a ribbon along which there is a distribution of centers of betatron oscillations. For this configuration, it is important to investigate in more details the consequences of space- 
charge effects. Also with only 350 turns circulating the beam peak current is 2.0-3.5 Amp in the $\mathrm{CW}$ mode of operation versus the 13-20 Amp in the pulsed mode. Correspondingly beam loading and RF power requirements are reduced.

Another advantage of the CW mode of operation is that beam is continuously injected and extracted thus simplifying the transfer. Moreover only positive ions, that are bare protons, are injected, and charge exchange at injection is not required.

\section{References}

[1] A.G. Ruggiero, "A 1-GeV 10-MWatt Proton Driver", Invited talk to ICFA-HB2004, October 18-22, 2004, Bensheim, Germany.

[2] M. Humbel et al., "Experience with and Theoretical Limits of High Intensity High Brightness Hadron Beams Accelerated by Cyclotrons", Invited talk to ICFA-HB2004, October 18-22, 2004, Bensheim, Germany.

[3] H. Padamsee, J. Knobloch. T. Hays, "RF Superconductivity for Accelerators", Wiley Series in Beam Physics and Accelerator Technology, 1998.

[4] J.S. Berg et al., "Review of Current FFAG Lattice Studies in North America", [5] L.C. Teng, "Linear Theory of Betatron Oscillations in Sectorial Cyclotrons", The Review of Scientific Instruments, Vol. 27, No. 12, Dec. 1956, pages 1051-1058.

[6] R.L. Kustom et al., "A 1500-MeV Fixed-Field Alternating-Gradient Synchrotron for a Pulsed-Spallation Neutron Source", IEEE Trans. on Nuclear Science, Vol. NS-32, No. 5, October 1985. 